Revue d'histoire de l'Amérique française

Q REVUE D.HISTOIRE DE L'AMÉRIQUE FRANÇAISE

STEVENSON, Garth, Ex Uno Plures: Federal-Provincial

Relations in Canada, 1867-1896 (Montréal et Kingston, McGill-Queen's University Press, 1993).44,95 \$

\title{
Christopher Armstrong
}

Volume 49, numéro 1, été 1995

URI : https://id.erudit.org/iderudit/305410ar

DOI : https://doi.org/10.7202/305410ar

Aller au sommaire du numéro

Éditeur(s)

Institut d'histoire de l'Amérique française

\section{ISSN}

0035-2357 (imprimé)

1492-1383 (numérique)

Découvrir la revue

Citer ce compte rendu

Armstrong, C. (1995). Compte rendu de [STEVENSON, Garth, Ex Uno Plures: Federal-Provincial Relations in Canada, 1867-1896 (Montréal et Kingston,

McGill-Queen's University Press, 1993). 44,95 \$]. Revue d'histoire de l'Amérique

française, 49(1), 108-110. https://doi.org/10.7202/305410ar d'utilisation que vous pouvez consulter en ligne.

https://apropos.erudit.org/fr/usagers/politique-dutilisation/ 
STEVENSON, Garth, Ex Uno Plures: Federal-Provincial Relations in Canada, 1867-1896 (Montréal et Kingston, McGill-Queen's University Press, 1993). 44,95\$

Ce ne sont pas les historiens qui se plaindront quand un chercheur annonce qu'il est retourné aux sources pour obtenir une meilleure compréhension de son sujet. Ceci est d'autant plus vrai lorsque le chercheur en question est un politologue qui traite d'un sujet historique et que l'application des théories des sciences sociales à des sources de première main, souvent complexes et contradictoires, a le potentiel de mener à des conclusions audacieuses. Attendu que notre compréhension du passé canadien a été enrichie par les travaux de politologues orientés vers des sujets historiques comme C. B. Macpherson et Richard Simeon, on ne peut que se réjouir de la décision de Garth Stevenson de réévaluer les relations fédérales-provinciales pendant les trente premières années de la Confédération.

Malheureusement, la consultation des sources ne garantit pas à elle seule des conclusions intéressantes et, à la lecture de Ex Uno Plures, on reste avec l'impression que les «trois années de recherches en archives» (p. $x$ ) n'ont pas abouti à un ouvrage que les spécialistes des relations fédérales-provinciales trouveront nouveau ou original. Stevenson déclare qu'il a adopté sa méthode parce qu'il trouvait que «la recherche historique, bien qu'elle éclaire les conflits entre le Dominion et les provinces, n'a pas réussi à analyser de manière satisfaisante l'impact mutuel des enjeux et des institutions ou la manière dont les conflits ont été abordés ou résolus» (p. ix). Si c'est vraiment le cas, il est curieux qu'il soit muet (ou du moins ne cite pas dans ses références) sur un nombre considérable de travaux historiques valables alors que les conclusions auxquelles il est parvenu divergent rarement de celles des chercheurs qui l'ont précédé.

Par exemple, il y a plus de dix ans, j'ai publié The Politics of Federalism: Ontario's Relations with the Federal Government, 1867-1942 (Toronto, University of Toronto Press, 1981), dont le premier chapitre 
discutait des trente premières années de la Confédération. Au moment de rédiger cet ouvrage, je savais que la thèse de J. C. Morrison, «Oliver Mowat and the Development of Provincial Rights in Ontario: a Study in Dominion-Provincial Relations, 1867-1896», rédigée en 1961, avait été publiée par les Archives de l'Ontario dans Three History Theses (s.l., s.d.). J'avais alors lu attentivement la thèse de Morrison, vérifié certaines de ses sources et conclu qu'il avait été diligent dans sa recherche et qu'il n'était donc pas nécessaire de refaire le même parcours que lui en lisant les collections de manuscrits comme les papiers d'Alexander Campbell. J'avais alors dirigé mes énergies ailleurs en consultant les études qui avaient suivi la thèse de Morrison ou le matériel inédit désormais accessible aux chercheurs. Je n'étais pas entièrement d'accord avec l'interprétation de Morrison sur l'époque car je trouvais que, comme son directeur de thèse Donald Creighton, il prenait trop le parti de John A. Macdonald aux dépens d'Oliver Mowat, mais j'ai certainement utilisé son matériel à bon escient. Le chapitre de Stevenson, «The Intergovernmental Agenda: Ontario» est sensiblement de la même longueur que celui que j'ai écrit sur la même période et les notes sont truffées de références à des collections de manuscrits (y compris les papiers de Campbell), mais ni le travail de Morrison ni le mien sont même mentionnés. Peut-être que l'auteur considère que nous sommes tous deux dans l'erreur, mais ce ne semble pas être le cas puisque Stevenson arrive presque aux mêmes conclusions, comme par exemple sur l'importance du conflit de personnalité entre Mowat et Macdonald qui est à la source du conflit entre les deux niveaux de gouvernement.

Cette faiblesse devient de plus en plus apparente lorsqu'on poursuit la lecture de l'ouvrage de Stevenson. Voici d'autres exemples. La loi de Mercier sur les biens des Jésuites est bien expliquée, mais il ne fait aucune mention de Equal Rights: the Jesuits' Estate Act Controversy (Montréal, McGill-Queen's University Press, 1979) de J. R. Miller. Il parle de la question des écoles du Manitoba mais sans faire référence à Priests and Politicians: Manitoba Schools and the Election of 1896 (Toronto, University of Toronto Press) de Paul Crunican. Évidemment, Stevenson n'est nullement tenu de citer qui que ce soit, mais ces deux monographies historiques sont solides et appuyées sur une lecture rigoureuse des sources. Si Stevenson se trouve à modifier ou à réfuter leurs conclusions, le lecteur a le droit de savoir comment et pourquoi, mais, en fait, ces études et d'autres sont laissées dans l'ombre pour faire la narration d'un sujet rebattu. Dans ce cas, qu'est-ce que la consultation des archives a vraiment donné?

Ex Uno Plures a une certaine utilité puisqu'il fournit une bonne synthèse des principaux débats entre le Dominion et les provinces et des rivalités intergouvernementales avant 1896. Si l'on cherche une description concise de la lutte menée par la Nouvelle-Écosse pour obtenir de «meilleures conditions» après la Confédération, on peut la trouver ici. Les étudiants de premier et de deuxième cycle en seront reconnaissants. Mais même dans cette optique, la structure du livre fait problème. Au lieu de retenir une classification thématique, Stevenson adopte une classification par région avec quatre chapitres sur les «enjeux intergouvernementaux» du Québec, de l'Ontario, 
des Maritimes et de l'Ouest. Cette stratégie est ambiguë étant donné qu'il doit mettre dans le même sac l'île-du-Prince-Édouard et les autres provinces de l'Atlantique, le Manitoba et la Colombie-Britannique, alors que l'auteur est le premier à admettre (en page 157) que les deux situations sont très différentes. Suivent cinq chapitres sur les structures (les réunions intergouvermentales, le rôle des lieutenants-gouverneurs, le désaveu, les relations anglocanadiennes et les décisions du Conseil privé). Par conséquent, des débats comme la Loi sur les biens des Jésuites et la question des écoles du Manitoba reviennent à plus d'un endroit (dans le survol par région, le désaveu, le Conseil privé, etc.), ce qui mène à une présentation quelque peu décousue. En théorie, le retour aux sources est une idée valable, mais Ex Uno Plures montre qu'en pratique, l'immersion dans les documents de l'époque ne suffit pas à elle seule à produire des résultats à la hauteur de nos espérances.

Département d'histoire 